\title{
A Review of Paper Currency Recognition System
}

\author{
Amol A. Shirsath ${ }^{1}$, S. D. Bharkad ${ }^{2}$ \\ ${ }^{12}$ Department of Electronics and Telecommunication Government College of Engineering, Aurangabad, \\ Maharashtra, India-431005
}

\begin{abstract}
This paper presents the review of paper currency recognition system. It is applied in various field such as foreign exchange, automatic selling of things and in banks. The recognition system mainly consists of three parts. The image of interest is first pre-processed by reducing dimensionality and extracting the feature by applying image processing toolbox of MATLAB, known as feature extraction. The second part is currency recognition, where classifier such as neural network is used. And lastly the result is displayed. Recognition ability depends on the currency note characteristics of particular country and extraction of features.
\end{abstract}

Keywords - Classifier, Currency recognition, Feature extraction, Foreign exchange, Preprocessing.

\section{INTRODUCTION}

Currency recognition is an image processing technology that is used to identify currency of various countries. Probabilities that the paper currencies of various countries are probably interweaved together therefore rises increasingly. It is a challenge for conventional paper currency recognition systems. However, the focus of most of the conventional currency recognition systems and machines is on recognizing counterfeit currencies. It is not enough for practical businesses. The reason is that in most of banks, especially those internationalized banks, there are large quantities of cash belonging to many different countries need to be process, and it is possible that all of them are real cashes. The situation that cashes belonging to different countries mixes together is possible to occur. It cannot be processed with conventional currency recognition systems.

Paper currency recognition systems should be able to recognize banknotes from each side and each direction. Since banknotes may be defaced during circulation, the designed system should have a meaningful accuracy in detecting torn or worn banknotes. The technology of currency recognition is used to research the visible and hidden currency characters, identify features all-around and dispose of the process on time. The original information has a loss because paper currency will get to the worm and blurry, even damaged by human being in circulation.

There are approximately 50 currencies all over the world, with each of them looking totally different. For instance the size of the paper is different, the same as the color and pattern. It is very difficult for persons who work in foreign exchange to distinguish currencies of different countries. Also paper currency became older than coins and also possibility of joining broken currency is greater than that of coin currency.

\section{Steps For PAPER CURRenCy ReCOGNition}

1. Image Acquisition

There are various ways to acquire image such as with the help of camera or scanner. Acquired image should retain all the features.

\section{Gray Scale Conversion}

Image is acquired in step 1 is large to continue process and colour information is not needed, except the colour index. First, RGB image is converted to pixel values and then to gray scale.

\section{Edge detection}

It is the fundamental tool in image processing, which aim at identifying points in digital image at which the image brightness changes sharply or has discontinuities.

\section{Image Segmentation}

It sub divides the image into its constituent regions. For monochrome images there are two basic properties of image intensity values. Similarity, in which image is partitioned into regions that are similar according to some predefined criteria. Other is discontinuity, in which image is partitioned based on abrupt changes in intensity for example edge. 


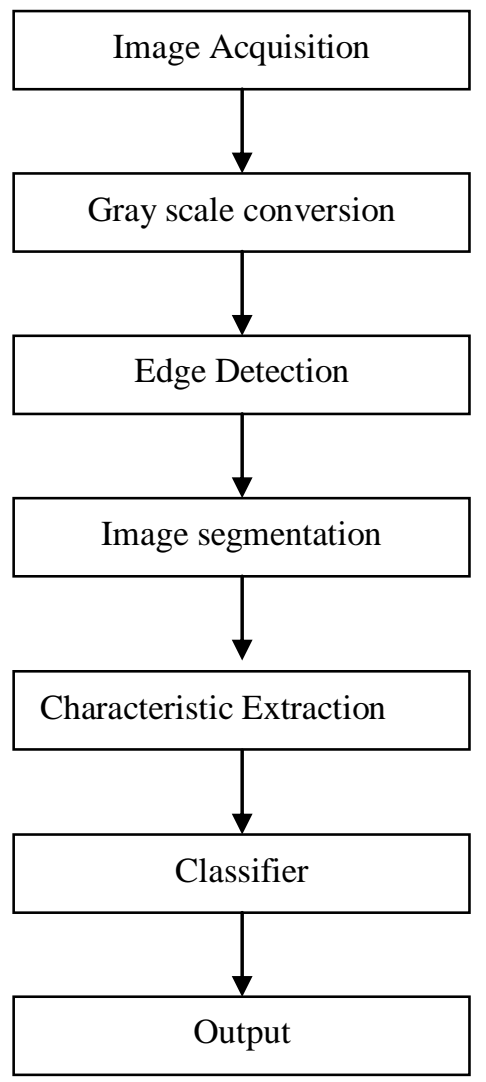

Fig.1 Steps of Paper Currency Recognition

\section{Classifier}

The input of the classifier will be the test currency images and the output of the classifier will be three parts:

1) The country name that using this currency;

2) The denomination of the input currency image;

3) The front or back side of the currency.

Neural network or Support vector machine can be used for training classifier.

\section{Output}

Output should be analysed for possibility of false rejection and false classification. By minimizing these two gives efficiency to the system. Output can be taken on hardware or on GUI (Graphical user interface).

\section{FEATURE EXTRACTION}

Feature extraction is a important procedure considerably for currency recognition, which effects on design and performance of the classifier intensively[1]. If the selected features are so large, it can easily construct a classifier with good recognition performance. The main task here is how to find effective feature among many features. There are mainly two types of features [1] as, Structural feature: It describes geometrical and topological characteristics of pattern by representing its global and local properties. Statistical Features: It describes characteristic measurements of the pattern.

In [2] component-based framework for banknote recognition by using Speeded Up Robust Features (SURF) is used. The component-based framework is effective in collecting more class-specific information and robust in dealing with partial occlusion and viewpoint changes. Furthermore, the evaluation of SURF demonstrates its effectiveness in handling background noise, image rotation, scale, and illumination changes.

Size, Colour, text these are the three characteristics of a currency used to distinguish between different banknote denominations.

\subsection{Size}

The first step of recognition of algorithm proposed by H. Hassanpour [3], considers size of the banknote. It is considered because during circulation of banknote worn and torn reduces its size, also it may increased slightly by rejoining torn banknote. Therefore they proposed decision tree as follows 


$$
\left|x-x_{0}\right|<d_{x} \&\left|y-y_{0}\right|<d_{y}
$$

Where $\mathrm{x}_{0}$ and $\mathrm{y}_{0}$ are size of the testing paper currency, $\mathrm{x}$ and $\mathrm{y}$ are size of reference paper currency. $d_{x}, d_{y}$ shows changes in the vertical and horizontal directions

\subsection{Texture Feature}

For texture feature LBP (Local Binary Pattern) operator [4] is used. Texture is the visible feature of the paper currency.

3.2.1 LBP operator: It is originally introduced by Ojala et al [4]. In LBP, the neighbourhood pixels are converted to binary code 0 or 1 by using the gray value of the centre pixel as threshold and further arranged to form as a ordered pattern. The feature extracted with LBP gives the relationship of the texture within local area. LBP code for pixel $p$ is defined as :

Where

$$
\operatorname{LBP}(p)=\sum_{i=0}^{7} 2^{i} s\left(g_{i}-g_{p}\right)
$$

$\mathrm{g}_{\mathrm{p}}-$ Gray value of the centre pixel $\mathrm{p}$.

$\mathrm{g}_{\mathrm{i}}-$ Gray value of the $\mathrm{i}^{\text {th }}$ pixel, 8-neighbourhood of $\mathrm{p}$

$\mathrm{i}-0,1 \ldots 7$.

$\mathrm{s}(\mathrm{t})=\mathrm{g}_{\mathrm{i}}-\mathrm{g}_{\mathrm{p}}$, is the threshold function and given by :

$$
s(t)=\left\{\begin{array}{l}
1, t \geq 0 \\
0, \text { else }
\end{array}\right.
$$

From equation 1 we can say that LBP can produce 256 kinds of different outputs, corresponding to 256 kinds of different binary patterns.

Junfang Guo, Yanyun Zhao[5] has proposed the improved method for texture analysis using LBP. They have segment the whole image into $\mathrm{M} * \mathrm{~N}$ blocks. In each block calculate the LBP value for every pixel, and make the histogram of the block, which is known as a block histogram. The block histogram is normalized by the number of pixels in the block.

3.2.2 Markov Chain Concept: As in many countries colour spectrum and size of some banknotes are very close to each other. For such a type of difficulties we are considering template of the banknotes. And to recognize these templates we are using Markov Chain Concept [6] to represent the random phenomenon.

A random process $\left\{\mathrm{x}_{\mathrm{k}}, \mathrm{k}=0,1,2 \ldots\right\}$ is called Markov chain if the possibility value in state $\mathrm{x}_{\mathrm{n}+1}$ depends on the possible value in state $x_{n}$, given as below

$$
\begin{aligned}
P\left(x_{n+1}\right. & \left.=\beta \mid x_{n}=\alpha, x_{n-1}=\alpha_{n-1}, \cdots x_{0}=\alpha_{0}\right) \\
& =P\left(x_{n+1}=\beta \mid x_{n}=\alpha\right) \quad(4)
\end{aligned}
$$

This possibility can be shown by $\mathrm{P}_{\mathrm{ij} \text {. }}$ The state space of a Markov chain can be shown in matrix as below

$$
P=\left[\begin{array}{cccc}
P_{11} & P_{12} & \ldots & P_{1 n} \\
P_{21} & P_{22} & \ldots & P_{2 n} \\
\vdots & \vdots & \ldots & \vdots \\
P_{n 1} & P_{n 2} & \ldots & P_{n n}
\end{array}\right]
$$

Where $\mathrm{n}$, is the number of states in the chain. In discrete time Markov chain, the possibility value of different states in the matrix is computed as

$$
P_{i j}=\frac{n_{i j}}{\sum_{k=1}^{n} n_{i k}}
$$

Where $\mathrm{n}_{\mathrm{ij}}$, is the number of transitions from state $\mathrm{i}$ to state $\mathrm{j}$. considering equation 5, matrix $\mathrm{P}$ can be multiplied by the denominator of equation 5 . To obtain

$$
P=\left[\begin{array}{cccc}
N_{11} & N_{12} & \ldots & N_{1 n} \\
N_{21} & N_{22} & \ldots & N_{2 n} \\
\vdots & \vdots & \ldots & \vdots \\
N_{n 1} & N_{n 2} & \ldots & N_{n n}
\end{array}\right]
$$


This is matrix is used to differentiate between textures in different denominations [4].

\subsection{Colour Feature}

If the primary image is in RGB format, then after resizing it is converted to HSV colour space[7]. Advantage of HSV colour space is that it is closer to human conceptual understanding of colours and has ability to separate chromatic and achromatic Components. Feature extraction of a colour image can be done by analysing its colour histogram, hue, saturation, intensity (or value)

In HSV (Hue, Saturation, Value) space hue distinguishes colour, Saturation is the percentage of white light added to a pure colour and value represents perceived light intensity. The transformation to HSV is achieved by following equations [8]:

\subsubsection{Conversion of colours from RGB to HSI:}

$$
\begin{gathered}
H=\left\{\begin{array}{cc}
\theta, & B \leq G \\
360-\theta, & B>G
\end{array}\right. \\
\theta=\cos ^{-1}\left\{\frac{\frac{1}{2}[(R-G)+(R-B)]}{\left[(R-G)^{2}+(R-B)(G-B)\right]^{\frac{1}{2}}}\right\} \\
S=1-\frac{3}{(R+G+B)}[\min (R, G, B)] \\
I=\frac{1}{3}(R+G+B)
\end{gathered}
$$

3.3.2 Conversion of colours from HSI to RGB: For RG sector:

For RB sector

$$
\begin{gathered}
0^{\circ} \leq H<120^{\circ} \\
B=I(1-S) \\
R=I\left[1+\frac{S \cos H}{\cos \left(60^{\circ}-H\right)}\right] \\
G=3 I-(R+B)
\end{gathered}
$$

For GB sector

For BR sector

$$
\begin{gathered}
120^{\circ} \leq H<240^{\circ} \\
H=H-120^{\circ} \\
R=I(1-S) \\
G=I\left[1+\frac{S \cos H}{\cos \left(60^{\circ}-H\right)}\right] \\
B=3 I-(R+G)
\end{gathered}
$$

$$
\begin{gathered}
240^{\circ} \leq H<360^{\circ} \\
H=H-120^{\circ} \\
G=I(1-S) \\
B=I\left[1+\frac{S \cos H}{\cos \left(60^{\circ}-H\right)}\right] \\
R=3 I-(G+B)
\end{gathered}
$$




\section{CLASSIFIER}

After getting features of currencies, it is essential to recognize the pattern of the currencies on the base of these features, which should be practised by an effective recognition system called classifier.

Fumiaki Takeda [9] proposed a new mask having symmetrical masked area against a axis dividing long side of paper currency in equal part, they called it as a symmetrical mask. Using this mask they have obtained same value from inverse and upright image of paper currency. Firstly geometrical meaning of symmetrical mask is shown and then procedure of the mask optimization by GA (Genetic algorithm) is given. Then neural network is constructed using optimized symmetric mask to recognize currency.

A Neural network based recognition scheme is used for Bangladeshi banknotes [10]. The scheme can efficiently be implemented in cheap hardware which may be very useful in many places. The recognition system takes scanned images of banknotes which are scanned by low cost optoelectronic sensors and then fed into a multilayer perception, trained by back propagation algorithm, for recognition.

Baiqing Sun [11] proposes a kind of currency recognition system, in which a three-layer feed forward neural network is used as a classifier, They proposed it in order to improve the performance of currency recognition system. In the feed- forward neural network a kind of Gaussian function is proposed as the activation function, which is employed in all units on the hidden layer and the output layer. The characteristics of this activation function are analyzed. It is a kind of localized function, its activation approaches zero as the distance to its centre approaches infinity. Its active region is governed by its width parameter. Just relying on this property of the proposed Gaussian, the rejection capabilities of the system can be improved.

As explained in [12] there are numerous models are developed to recognize paper currency. Such as auto associative network, nonlinear auto associative network, radial basis function (RBF) network. Most of them uses single neural network with one hidden layer but there are certain limitation of this networks. Firstly, single $\mathrm{NN}$ is not sufficient to train all aspects of currency note. Secondly, decision was made completely from a NN and not from a group of NNs.

A comparision of various method with each other is tabulated in TABLE I in terms of recognition rate. Each method uses different paper currencies.

TABLE I

COMPARISION OF VARIOUS METHODS

\begin{tabular}{|c|c|c|}
\hline Methods & Cureency & $\begin{array}{c}\text { Maximum } \\
\text { Recognition (\%) }\end{array}$ \\
\hline HMM [3] & Various & 95 \\
\hline ENN [13] & Bangladesh & 100 \\
\hline RBF [14] & Euro & 100 \\
\hline SLCRec [15] & Sri Lanka & 100 \\
\hline
\end{tabular}

\section{CONCLUSION}

This paper discussed a feature extraction technique for paper currency. Depending on its size, texture and colour. Techniques include Markov chain concepts, Local binary pattern technique. Also paper explains general block diagram for currency recognition system, it includes various steps starting from image acquisition and at the end of classifier step result will be shown. Classifier discussed in this paper is neural network employing guassian function. The proposed work is effort to present review of paper currency recognition system.

\section{REFERENCES}

[1] Parminder Singh Reel, Gopal Krishan, Smarti Kotwal, Image Processing based Heuristic Analysis for Enhanced Currency Recognition, International Journal of Advancements in Technology, Vol 2, No 1, January 2011.

[2] Faiz M. Hasanuzzaman, Xiaodong Yang and Yingli Tian, Robust \& Effective Component based Banknote Recognition by SURF Features, NSF grant IIS-0957016.

[3] H. Hassanpour, A. Yaseri, G. Ardeshiri, Feature Extraction for Paper Currency Recognition, IEEE Transactions, 1-4244-0779-6/07, 2007.

[4] T Ojala,M Pietikainen, and D Harwood, A comparitive study of texture measures with classification based on feature distributions, Pattern recognition, Vol.29, No. 1, 1996, pp.51-59.

[5] Junfang Guo, Yanyun Zhao, Anni Cai, A Reliable Method for Paper Currency Recognition Based on LBP, IEEE Transactions, Proceedings of IC-NIDC2010, 978-1-4244-6853-9/10.

[6] M. Iosifescu, Finite Markov Processees and Their Applications, Wiley, New York, NY, 1980.

[7] G. Trupti Pathrabe, Mrs. Swapnili Karmore, A Novel Approach of Embedded System for Indian Paper Currency Recognition, International Journal of Computer Trends and Technology- May to June Isue 2011, ISSN: 2231-2803.

[8] Rafael C. Gonzalez, Richard E. Woods, Steven L. Eddins, Digital Image Processing Using MATLAB,2012.

[9] Fumiaki Takeda, Toshihiro Nishikage, and Yoshiyuki Matsumoto, Characteristics Extraction of Paper Currency using Symmetrical Masks Optimized by GA and Neuro-Recognition of Multi-National Paper Currency, IEEE Transactions, 0-7803-4859-1/98, 1998.

[10] Nadim Jahangir, Ahsan Raja Chowdhury, Bangladeshi Banknote Recognition By Neural Network with Axis Symmetrical Masks, IEEE Transactions, 1-4244-1551-9/07. 
[11] Baiqing Sun, Research on Rejection Capabilities of Paper Currency Recognition System with the Neural Network Employing Guassian Function, Kochi University of Technology, Japan,2006.

[12] Masato Aoba, T. Kikuchi, Y. Takefuji, Euro Banknote Recognition System using a three layer perceptron and RBF networks, IPSJ transaction on Matematical Modeling and its Applications, vol 44, 7(8), pp. 99-109, 2003.

[13] Kalyan Kumar Debnath, Sultan Uddin Ahmed, Md. Shahjahan, A Paper Currency Recognition System Using Negatively Correlated Neural Network Ensemble, Journal of Multimedia, vol. 5, No. 6, December 2010.

[14] F. Takeda and T. Nishikage, Multiple kinds of paper currency recognition using Neural Network and application for Euro currency, In Proc. IEEE International Joint Conference on Neural Networks, pp 143-147, 2000.

[15] D. A. K. S. Gunaratna, N.D. Kodikara and H. L. Premaratne, ANN Based Currency Recognition System using Compressed Gray Scale and Application for Sri Lankan Currency Notes-SLCRec, Proceedings of world academy of science, engineering and technology, vol. 35, ISSN 2070-3740, Nov 2008. 